\title{
Study on Nonlinear Friction-Induced Vibration in Water-Lubricated Rubber Stern Tube Bearings
}

\author{
Engao Peng ${ }^{*}, 1$, Zhenglin Liu ${ }^{1}$, Xincong Zhou ${ }^{1}$, Yuzhong Tian ${ }^{2}$, Meiyun Zhao ${ }^{1}$ \\ ${ }^{1}$ School of Energy and Power Engineering, Wuhan University of Technology, Wuhan 430063, China \\ ${ }^{2}$ Wuhan Rule and Regulation Research Institute, China Classification Society, Wuhan 430022, China
}

\begin{abstract}
Nonlinear friction-induced vibration deteriorated the performance of marine rubber stern tube bearings (RSTB) in a wide variety of propulsion system. In the paper, this study was performed on the level of both practical and experimental bifurcation analysis. Hereto, an experimental set-up of SSB-100 marine stern tube bearing test machine was applied to carry on this project. The synthesis of these practical and experimental results confirmed that nonlinear frictioninduced vibration was due to a subtle balance of negative damping at lower speeds or viscous friction at higher speeds. Furthermore, it was shown how these essential friction characteristics depended on physical conditions such as temperature, normal forces and the material properties in the frictional contact.
\end{abstract}

Keywords: Water lubrication, stern tube bearings, friction-induced vibration, squeal, noise.

\section{INTRODUCTION}

Vibration and noise referred to friction-induced vibration that occurred as the result of energy loss from propulsion system. There were a number of sources of vibration and noise existing in a ship or marine vehicle. The frictioninduced vibration of the RSTB easily caused low-frequency quaver and high-frequency squeal in a marine. Though it could be shown that the majority of the mechanical energy from a marine was converted to kinetic energy or heat, a partial still was converted directly into vibration that could be heard or felt by the operator. This test showed that under conditions of certain temperature and rotational speeds, the friction coefficient was increasing along with the load increasing if the contact state of the RSTB was unsaturated elastic. The water-film elastic hydrodynamic lubrication formed at high rotational speeds. But under conditions of low speed, heavy load, partial medium temperature and bad lubrication, abnormal vibration and squeal occurred unexpectedly. These seriously influenced the invisibility and survival ability of the marine.

Wear, friction coefficient and noise were considered to be the most critical elements of water-lubricated stern tube bearings' performance [1]. Therefore, a number of investigations related to the tribological properties of the RSTB had been carried out and the friction and wear performance of the RSTB had been comparatively concluded that the rubber had well embedding properties. What's more, the RSTB had better wear resistance in the sediment water than that of other bearings [2]. T.A. Simpson and R.A. Ibrahm [3] extensively studied the problem of frictioninduced vibration and squeal in water-lubricated shipboard

*Address correspondence to this author at the School of Energy and Power Engineering, Wuhan University of Technology, Wuhan 430063, China; Tel/Fax: +86-027-50241480; E-mail: 863631723@qq.com bearings. Their studies were dominated by experimental tests of section models that emulated the actual bearing dynamics. And linear analytical models were analyzed to predict the stability boundaries of the equilibrium position. Different dynamic characteristics were predicted from the numerical simulation of the equations of motion. A bifurcation diagram was constructed and exhibited different regimes. The squeal was revealed by the presence of high frequency vibration superimposed on the fundamental system frequency. The occurrences of each regime mainly depended upon the value of slope of the friction-speed cure.

Bharat Bhushan [4] developed a fundamental understanding of the mechanism of noise generation in water-lubricated compliant rubber bearings. The study had clearly demonstrated that the basic phenomenon was a stickslip notion of the rubber surface occasionally coupled with the mechanical resonances of the bearing parts. Forever, the squeal was a vibration and noise phenomenon that was generated by a stick-slip motion in the rubber at the surface where it contacted the shaft. The squeal was encouraged by a roughened elastomeric surface.

H.T. Banks [5], J.B. Hood, et al. designed a rouse model for polymer chains, which was incorporated into the linear continuous stick-slip molecular-based tube reputation ideas of Doi-Edwards and Johnson-Stacer. This yielded an explicit system of stress-strain equations for the system permitting simple calculations of complex stress-strain relations. The viscoelasticity of polymers was studied in the contest of dilute solutions. The molecular-based applicable model sought to describe hysteresis in these elastomers. The model was direct, more rigorous and brought together an idea that was largely phenomenological, which was nevertheless plausible from energy and thermodynamics considerations. Based on a linear stick-slip assumption, the model captured a considerable amount of the hysteresis in several types of materials, but it didn't capture especially well the shapes of 
the nonlinearities in the hysteresis loops. Further development along the lines of the nonlinear stick-slip assumptions was needed while the model was useful initial effort.

Dae Kyun Baek and M.M. Khonsari [6, 7] presented friction characteristics of a rubber coating undergoing fretting motion. The friction coefficient did not reach the maximum value right after the rubber coating failed, but remained low for a long time because the rubber debris acted like a solid lubricant. This property could enhance fretting fatigue life and reduce fretting wear [8]. The friction coefficient of the rubber coating reached the maximum value as the load increased and as the displacement amplitude increased too. The rubber coating life was related to the running conditions, i.e. the load, the velocity and the displacement amplitude. When the humidity and temperature were held constant, these factors were not considered though they might also play a small role. And the coating life was found to be very short when the system underwent oscillatory motion in a low velocity regime. The wear of the rubber seemed to be related to the total running time at low velocity and to be related to relevant adhesion.

Roy L. Orndorff, Jr [9] developed a new polymer bearing alloy that overcame the high wear non-hydrodynamic deficiencies of UHMWPE and the shaft wear and high breakaway friction of rubber bearings when working in water-lubricated conditions. A number of competing materials were also wear tested with the new alloy comparing favorably. It discovered that the rubber bearing wear increased under these conditions. It was hypothesized that the higher-pressure clean-water test sample of the new alloy adjusted to run in the hydrodynamic regime though elastic deflection, creep and a minimal amount of wear. The coefficient of friction versus shaft (journal) speed curve decreased in the mixed and hydrodynamic regimes with increasing load. As shown in Fig. (1), the classical friction curve reflected the changes of the friction coefficient.

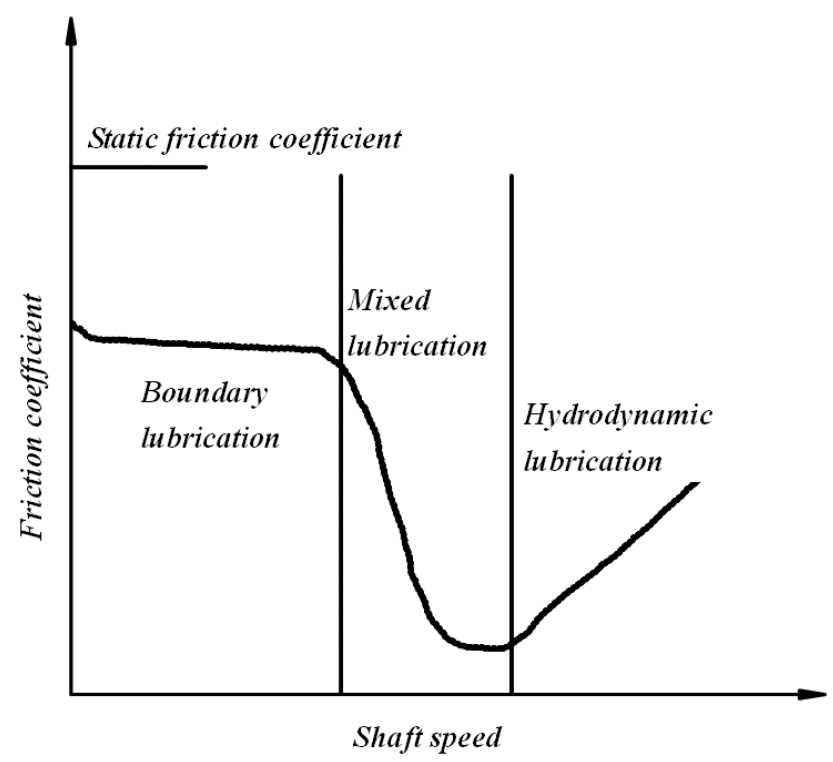

Fig. (1). Classical friction curve.

\section{EXPERIMENTAL DETAILS}

It was previously thought that better modeling of the phenomenon of hysteresis would lead to better understanding of the conditions required for the onset of vibration and squeal [10]. Notwithstanding it seemed that while hysteresis affected the nature of the stick-slip induced vibration, it was possible to see more than one type of dynamical behavior for the given system. A phenomenological model was proposed which combines the concepts of static and dynamic friction. This seemed intuitively reasonable and for which there was documented evidence to verify what the above said [11]. In the test the vibrating structure was simplified as that of a rotating shaft resting on the RSTB, axial restrained by two rolling bearings. The nonlinear system dynamics predicted by applying the new friction model were unusual in that the conditions giving rise to squeal including not only the rotational speed of the shaft, but also the initial conditions of the RSTB structure.

\subsection{Model of the Water-Lubricated RSTB}

The external diameter of the RSTB was about $170 \mathrm{~mm}$, the inner diameter was nearly $149 \mathrm{~mm}$ and the length was $150 \mathrm{~mm}$. The length-to-diameter ratio was 1:1. There were equally distributed 10 water flumes which were $8 \mathrm{~mm}$ width and $6 \mathrm{~mm}$ depth respectively along the inner wall of the bearing. The working surface of the strip was plane and there was slightly a little chamfering around the corner, as shown in Fig. (2).

\subsection{Test Rig}

The vibration test of the RSTB was conducted by the SSB-100 marine RSTB test machine, which was developed by the stern tube bearing lab of Wuhan University of Technology. The test machine was mainly composed of a driving system, a test system, a load system and a measurement system, as shown in Fig. (3). The shaft was made of 45\# steel and a bronze (ZQSn10-2) bushing block embedded in the journal. The length of bushing block was $175 \mathrm{~mm}$ and its external diameter was $150 \mathrm{~mm}$. There were additionally a water lubrication system which could adjust the tap water flow and a heating system which could adjust the water temperature according to the test requirements.

\subsection{Test Procedure}

The measurement system was composed of a tachotorque meter, a pressure meter, a flowmeter and a BK (pulse) vibration test device. The tacho-torque meter was applied to measure friction torque, which could be converted to friction coefficient. The pressure meter was applied to record oil pressure, which could be converted to specific pressure. The flowmeter was applied to measure the water flow, which could be estimated for lubrication. And the BK (pulse) vibration test device was applied to extract and analyze the vibration signals, which were the reflection of vibration and squeal.

The RSTB were fixed to the bush and a pre-stress was applied to the ring at vertical direction. The testing load, which was supplied by hydraulic pump, was brought to the 

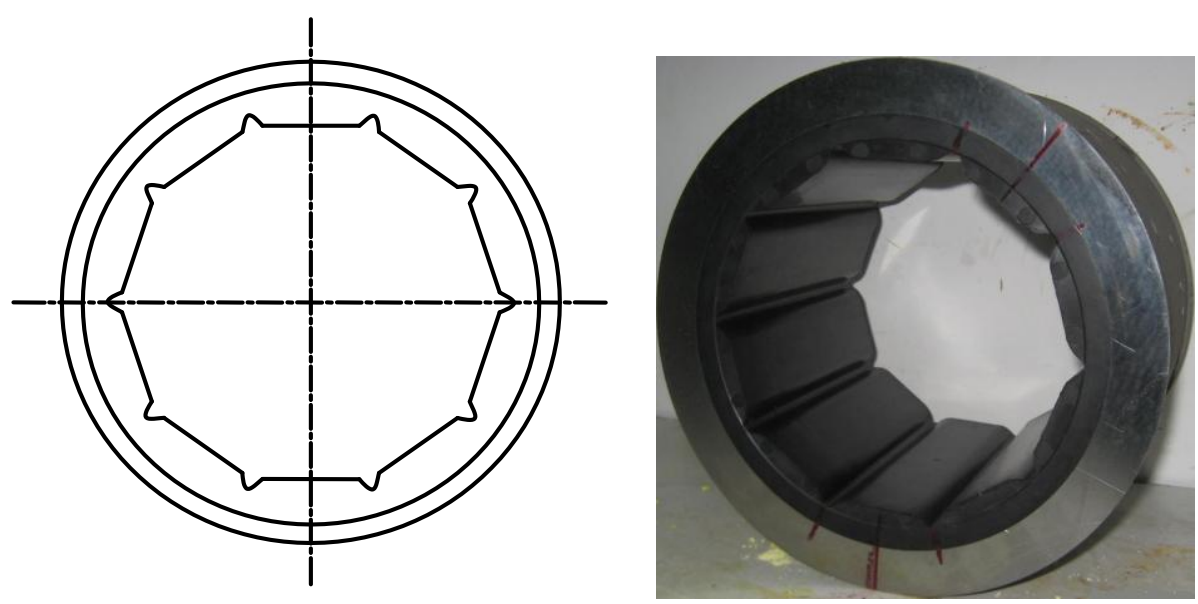

Fig. (2). Diagram of RSTB model.

RSTB at the intermediate position. In the operation process of the test machine, the testing load was converted to the form of the specific pressure. Then the variation of the specific pressure synchronously represented the variation of the testing load. There a rectangular contact regime was formed between the RSTB and the shaft. After the contact regime was stable in a short time, a certain rotational speed was applied to the shaft. After the rotational speed of the shaft was stable for a period of time, the operator could survey the vibration at startup. Conversely, the operator also could survey the vibration when the test machine suddenly stopped. Furthermore, signals of vertical and horizontal spectrum were measured and analyzed simultaneously. The friction coefficient of the rubber sample was measured automatically and displayed synchronously by the test computer monitors.

\section{VIBRATION TEST OF WATER-LUBRICATED RSTB}

In the vibration test of the water-lubricated RSTB, rotational speed, temperature and specific pressure were being controlled in an appropriate range. The rotational speed was within $800 \mathrm{r} \cdot \mathrm{min}^{-1}$ and the specific pressure was less than 0.5 $\mathrm{MPa}$. Because of the loading regime of intermediate load, the vibration test points were strictly chosen on the outer ring of the middle section of the RSTB in horizontal and vertical directions. The locations of the sensors were vertical at the intersection of the axial and could be seen in Fig. (3).

\subsection{Friction Coefficient-Speed Characteristics Test}

In the process the rotational speed varied from low to high. With the speed increasing slowly, the lubricating conditions ameliorated gradually and the contact area increased simultaneously. Then the adhesive friction coefficient decreased firstly, near the inflection point it increased slowly for a while and almost unchanged along with the increasing of speed at a limited speed. According to the results, it could be concluded that the friction coefficient had a negative correlation to do with the rotational speed and the inflection point was appeared in low-speed area, as shown in Fig. (4). In these areas, the friction coefficient was stable and changed little, but there was extremely slow growth trend. At a limited speed, the friction coefficient increased along with the increasing of temperature. Similarly, when the friction coefficient was fixed, the temperature increased along with the increasing of rotational speed. In addition, the inflection point of the vibration and squeal appearing located in low speed area.

Under these conditions, the operating status of the RSTB behaved unstable along with the change of the working conditions. The vibration and squeal occurred sensitively

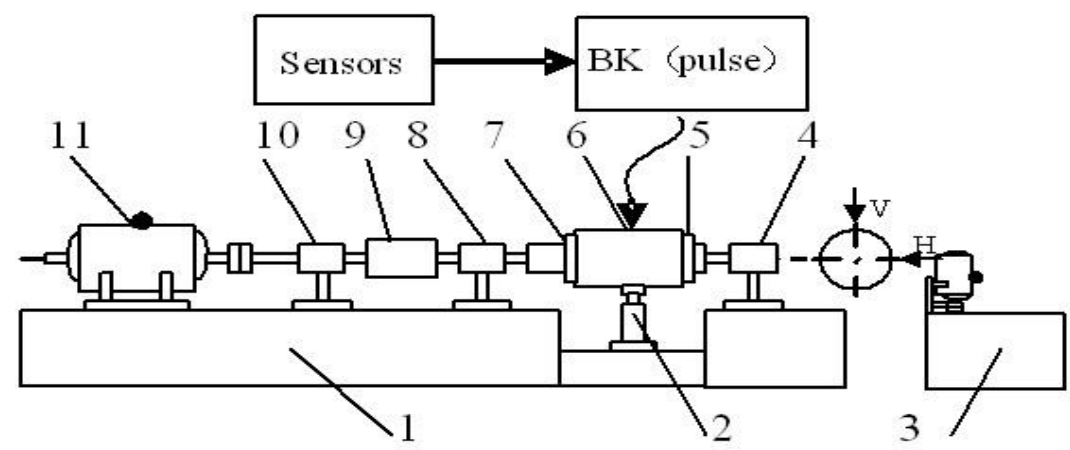

1-base; 2-hydraulic jack; 3-hy draulic pump; 4,8,10-supports; 5,7-face seal;

6-RSTB; 9- tacho-torque meter;11-variable frequency motor

Fig. (3). SSB-100 marine RSTB test machine. 
when there was a little incentive, which verified the randomness of the vibration and the radiation of the squeal. These could be reflected by the time-frequency signals acquired accompanying the vibration and squeal test. The test conditions and results were shown in Fig. (5), where the critical speed of the squeal varied with the change of the specific pressure at different temperature. At a fixed temperature, the higher the specific pressure was, the higher the rational speed was while the vibration and squeal occurred. Similarly, when the rational speed was fixed, the higher the temperature was, the higher specific pressure was while the vibration and squeal occurred. The properties achieved stable rotating conditions at a rated rotational speed. And water was also utilized to restrain the probability of vibration and squeal occurrence through water heat dissipation and lubrication effect.

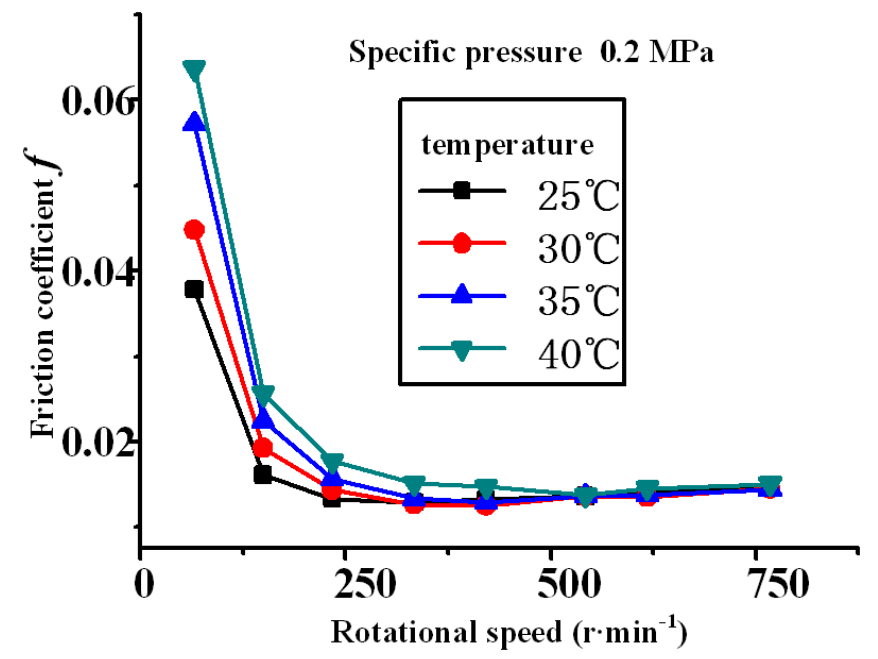

Fig. (4). Friction coefficient-speed characteristics cure.

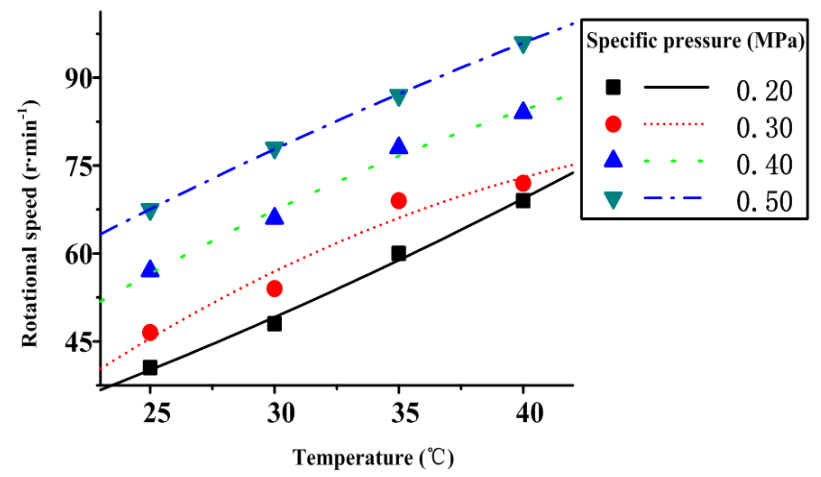

Fig. (5). The critical speed of squeal occurring.

\subsection{Vibration and Squeal Test}

The nature of squeal was high-frequency vibration. Through squeal maps acquired by pulse vibration test device we could analyze how the vibration and squeal occurred. As the rotational speed varied from low to high, vibration and squeal phenomena became significant. Figs. $(6,7)$ showed the squeal maps of with or without squeal under the conditions of $35^{\circ} \mathrm{C}$ and $0.40 \mathrm{MPa}$.

In the time domain, no-squeal signals of the bearing were kept stable almost all the time. The amplitude presented to be stripped and the maximum peak-to-peak value was less than $0.04 \mathrm{~m} \cdot \mathrm{s}^{-2}$, which was the reflection of the tacho-torque meter, as shown in Fig. (6a). These might be synthetically caused by machine natural vibration, motor vibration, aqueous medium eddy [12] and hydraulic pulse excitation. Squeal signals had greater changes and the enveloping line of the amplitudes had periodic ups and downs which was similar to rotational frequency. The wave formed inside the enveloping line had a strong nonlinearity and the maximum peak-to-peak value was $0.20 \mathrm{~m} \cdot \mathrm{s}^{-2}$, as shown in Fig. (7a). This might be the effect of the jerk dynamics [13] on the inner surface of the bearing and the nonlinear frictioninduced vibration.

In the frequency domain, no-squeal signals of the bearing spectrum changed invariably and higher amplitudes mainly focused in low-frequency region. It was affected by rotational frequency and impulsion of hydraulic system, whose maximum peak was around $0.25 \mathrm{kHz}$, as shown in Fig. (6b). Squeal signals spectrum changed a lot and obviously higher amplitudes mainly focused in highfrequency region, as shown in Fig. (7b). Basic frequency of high-frequency was around $1.25 \mathrm{kHz}$. These peak points differed from each other nearly by a magnitude of $1.25 \mathrm{kHz}$ in the spectrum. When squeal occurred, the incentive was still the unbalanced rotational movement of the shaft and that of hydraulic system [14], which indicated the vibration response having the nature of randomness.

The experimental results showed that the vibration of the bearing was induced by friction. When the vibration approached the generation frequency, the response enhanced firstly and weakened gradually along with the fading of the vibration under the working status of increasing speed, which meant there being a negative correlation between vibration and rotational frequency. The RSTB limitations under usage conditions were established as result of vibration test done on the bearings and load tests on the rubber at a rated rotational speed of less than $800 \mathrm{r} \cdot \mathrm{min}^{-1}$. The operation tests on the RSTB were carried out under a specific pressure of $0.5 \mathrm{MPa}$ and a temperature of $40{ }^{\circ} \mathrm{C}$, which revealed that the operation had significant effects on increased onset of vibration and squeal. However, the normal vibration of the rubber influenced the slip velocities and the distribution of frictional forces [15]. In a microscopic range the harmonically oscillating long cylinder sliding on the flat surface of an elastic substrate of the rubber, the time histories of the width of the contact zone and the length of adhesion subzone were partial. The local frictional forces and slip velocities oscillated, providing the rolling/sliding regime was smaller under conditions of normal vibrations than that when the frequency was not near to the generation frequency and the RSTB did not vibrate distinctively yet. The frequency changed in time-frequency presentations were associated with nonlinearity of vibration systems. The nonlinearity might be counted as the evidence to support the consideration that friction-induced vibrations were bounded by limit cycles due to the system nonlinearity. Based on the time-frequency presentation of vibrations, it could be concluded that the friction-induced vibration system was generally a linear system in the stage of vibration initiation while was nonlinear system in the stages of vibration bounded and fading [16]. The friction contact change at the interface contributed to the generation of nonlinear frictioninduced vibration. This test could quite effectively identify the occurrence of vibration and squeal in the time domain 


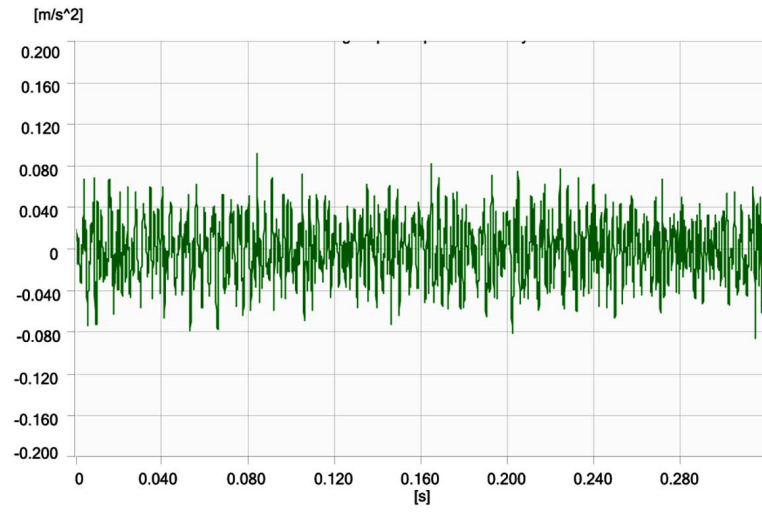

(a) Time domain signals without squeals

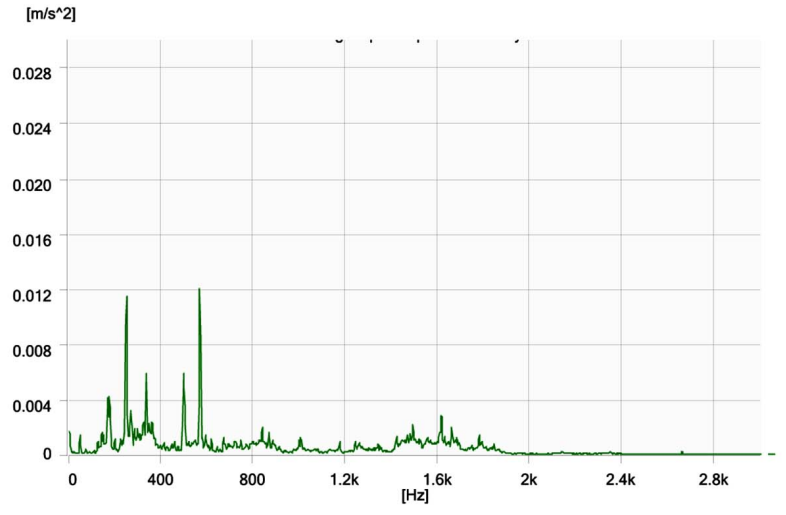

(b) Frequency domain signals without squeals

Fig. (6). Time-frequency characteristics curves without squeals $\left(0 \mathrm{r} \cdot \mathrm{min}^{-1}\right)$.

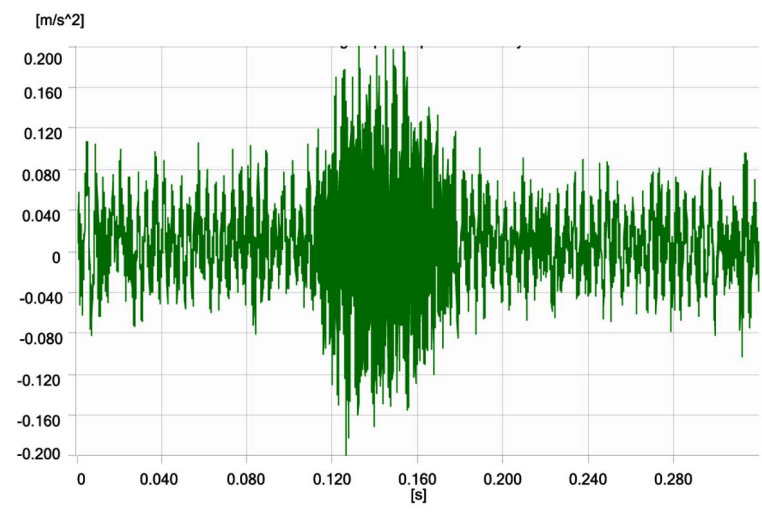

(a) Time domain signals with squeals

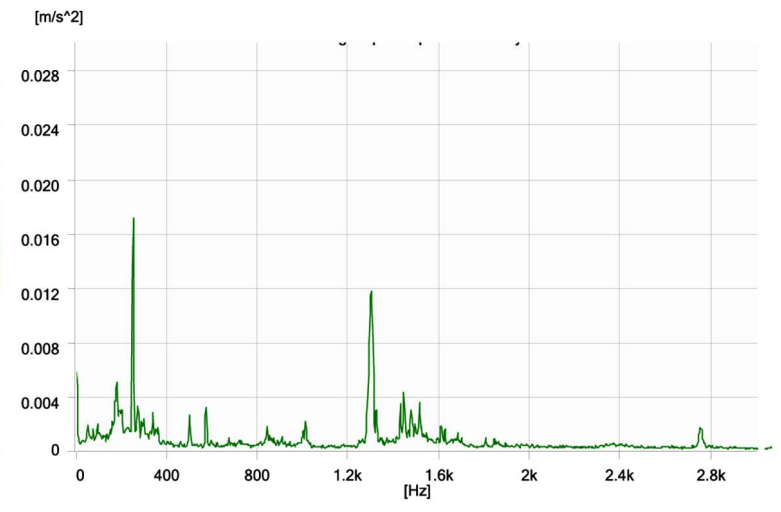

(b) Frequency domain signals with squeals

Fig. (7). Time-frequency characteristics curves with squeals $\left(22 \mathrm{r} \cdot \mathrm{min}^{-1}\right)$.

provided responses were adequately separated and strong enough.

The eccentric operation of the shaft caused the RSTB to vibrate and radiate noise [17]. The squeal radiation from the RSTB in the low frequency range might be the fluctuating forces at the propeller and these forces were transmitted to the RSTB via the shaft and the fluid [18]. These were correlated with a loss of dynamic instability [19]. Because of no-centering and imbalance of the shaft, the RSTB got energy supplement when vibration occurred and generated periodic response synchronously. In some extreme conditions such as starting/stopping stages a little damping would cause the system to have several times of response or large changes.

\section{NONLINEAR FRICTION-INDUCED VIBRATION CAUSES ANALYSIS}

The vibration causes varied from different types of materials, and there was something to do with the working conditions. But the primary causes were not beyond two main ones, including dynamics stability and dynamics instability. When the RSTB were being operated, there were complex dynamics issues that needed to be solved and to be perfected.

\subsection{Dynamics Stability Analysis}

There were mainly two reasons for the friction-induced vibration of the water-lubricated RSTB. Firstly, it was exterior damping effect. There were water film and hydrodynamic lubrication in local area. The water film played two roles. It reduced the friction and lubricated the shaft. Moreover it hindered the normal operation of the shaft and brought forth tangential viscous force. Simultaneously there was air damping in the seal cavity. When the hydraulic pressure was stable, partial air dissolved in water and partial air discharged outside along with the running water. Secondly, it was internal damping effect. In the process of friction-induced vibration the elastic-plastic deformation of the rubber absorbed and released energy. There was energy loss in this switching process. This kind of energy loss and the vibration frequency was closely related to each other [20].

The frequency was higher and the dynamic stiffness of the RSTB was smaller, then there was no enough time for compression deformation occurring and the expenditure of energy was smaller too. The frequency was lower and the dynamic stiffness of the rubber was larger, then the energy absorption and release process completed without enough time. At the moment based on the friction-induced vibration 
it possibly consumed energy. Thus the mechanical energy transformed into kinetic energy or heat. It could be verified that the temperature of the outlet water was higher than that of the inlet water. Although the internal damping didn't play a leading role of energy consumption, it could gradually corrode the driving force when the RSTB were operated at low speeds, under heavy load and at medium temperature.

Adopting the method of eigenvalue analysis, it could predict the vibration and squeal of the bearing system. According to the linear theory, when squeal occurred, the vibration amplitude of the bearing would get larger gradually till the bearing system failed. But the practical truth was that the vibration corresponding to the frictional noise was bounded. On the direction of sliding that the rubber surface occurring elastic-plastic deformation there was easy to generate squeal along the direction of the frictional force [21]. Therefore, in order to avoid the obvious level of noise or squeal, the shaft speed should be constrained at a limited speed, as what the paper had mentioned above.

The stress performance of the RSTB was very complex. Besides the quality of the stern shaft and the propeller, there were inertial forces that caused by the imbalance quality of the propeller and additional forces that caused by adverse shafting alignment plus the hull deformation [22]. Conversely, the vibration response of the RSTB influenced the vibration characteristics of the marine shafting.

\subsection{Dynamics Instability Analysis}

At low speeds the internal structure of the rubber was out of shape, the water film didn't form and the lubrication conditions were deteriorated. At the moment the RSTB were under mixed friction conditions and in mixed lubrication, even in local area there may be in dry friction conditions yet. And it was possible to have pressure water-pocket effect in the local area [23, 24]. Both the wobbling mode of the shaft and the elastic deformation mode of the RSTB had much to do with the friction coefficient, temperature and specific pressure. And we could alter the coupling with the wobbling mode.

This complex instability and its effect were correlated with experimental observations. Under the effect of water pressure and load, the partial pressure got higher and the rubber surface was compressed to form water-pocket in local area. Then the interior water in the water-pocket bladders and the exterior water were isolated of convection. As shown in Fig. (8). In a very short time the stick-slip movement came into being and the water pressure was released. Plus resilience characteristics of rubber it resulted in an expansion that impeding the normal operation of the shaft. And then the shaft departed from its dynamic equilibrium position. This caused vibration which was namely obvious friction-induced vibration phenomenon familiar to all. The speed was called critical speed. Because the speed was varied and the operating conditions were unstable, the vibration was nonlinear yet. Simultaneously, unusual squeal occurred and the vibration got disorder according to the varied actual operating mode. In a limited speed, the vibration and squeal or noises still existed only if the shaft returned to the dynamic equilibrium position and the lubrication condition was improved upon hydrodynamic lubrication.

At the same time this kind of friction-induced vibration possibly caused the rubber and the metallic material immediately to contact friction fiercely and dissipated heat. A lot of heat energy couldn't dissipate promptly in a short time and contributed to the localized high temperature. It caused the rough peak of the rubber surface and the surface of the shaft to have viscous force because of the intermolecular force. Though this phenomenon behaved as a negative damping effect to the shaft, it played an actuation role to the RSTB $[25,26]$, which caused the intermittent vibration. The viscous force destroyed theirs equilibrium since the RSTB were constrained. These added external and internal damping respectively, which led corresponding responses to unstable status and dominated the system response. Here squeal occurred inevitably.

Though water was a nearly Newtonian fluid, it followed Newton's viscosity rule. The density and viscosity of the water changed slightly with the pressure and temperature $[27,28]$. Thus this dynamics instability could destroy the machining accuracy of the working parts. Adversely, the failure of key components affected the normal operating of the RSTB [29, 30]. As a result, this viscous circle accompanied with nonlinear manifestations mode and abnormal vibration or squeal occurred naturally. As shown in the time-frequency characteristic cures with squeals mentioned above.

To sum up, it could get the nonlinear friction-induced vibration causes of the water-lubricated RSTB. When the
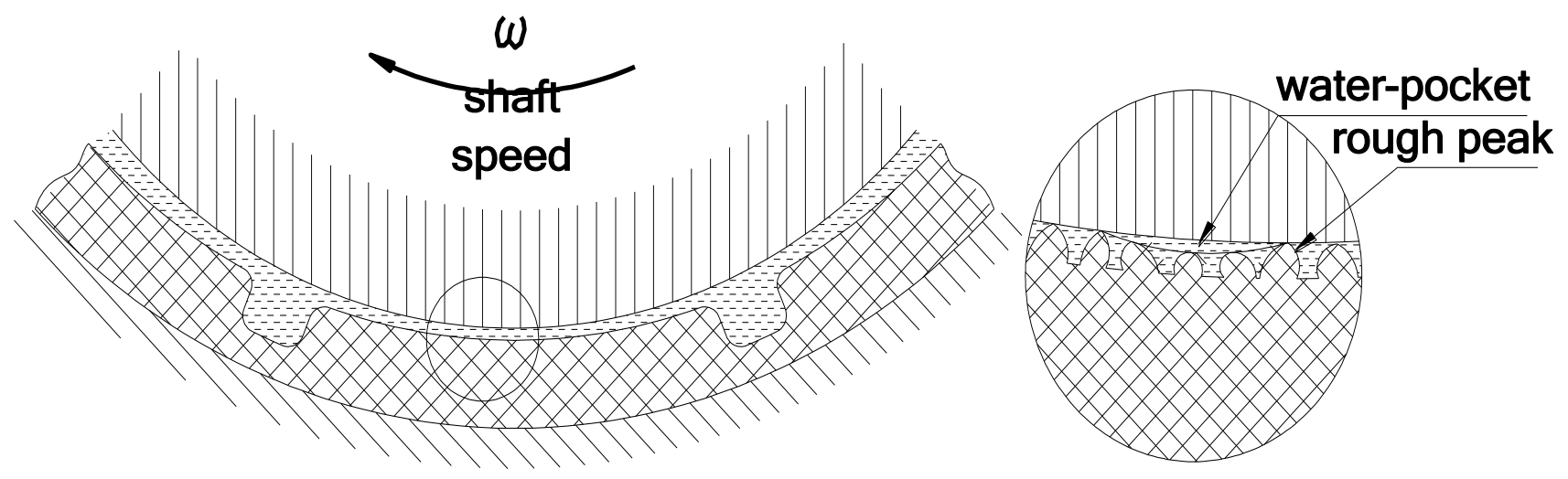

Fig. (8). The viscosity role of rough peak on the rubber surface. 
shaft rotated, it contacted with the rubber and the dynamic stiffness of the structure increased. Then the RSTB deviated from the shaft after the rubber restored and the dynamic stiffness of the structure decreased. The dynamic stiffness of the structure was varied in this time quantum. At the moment the static friction force got larger than the dynamic friction force. The relative movement between the shaft and the bearing possibly formed the intermittent movement. This destroyed the equilibrium state of the friction components, and then the friction-induced vibration occurred. In essence, this vibration was self-excited vibration. This vibration caused severe wear to the RSTB, destroyed the size of the key components, reduced the system efficiency and radiated noise, which easily formed noise pollution. When the shaft was being operated at low speeds runtime, this kind of phenomenon was widespread and inevitable.

\section{SUMMARY AND CONCLUSION}

This paper presented a method focused on experimental study of nonlinear friction-induced vibration on the modeling of friction effect, which were realized by means of friction elements placed in the shaft and the RSTB. Combined with theoretical analysis and experimental study, the nonlinearity was due to the friction-induced vibration and was modeled based on experimental measurements. The nonlinear response of the coupling vibration was responsible for the occurrence of squeal or noise. In addition, we showed that the level of negative damping in the friction at lower speeds in relation to the level of viscous friction appearing for slightly higher speeds determined whether nonlinear friction-induced vibration with or without stick-slip could appear. In a limited range the vibration got weaker when the rotational frequency increased and got stronger when the rotational frequency decreased. And the direction of the stick-slip had important influence on the occurrence of squeal for the frictional force caused the elastic-plastic deformation of the rubber surface along that direction. And the diversity of the friction state contributed to the generation of friction-induced vibration nonlinearity.

\section{CONFLICT OF INTEREST}

The authors confirm that this article content has no conflict of interest.

\section{ACKNOWLEDGEMENTS}

This research was supported by National Natural Science Foundation of China (No.50979084 and No.51079119), Key Natural Science Foundation of Hubei Province (No.2008 CDA027), and Innovation Research Fund of Wuhan University of Technology (2010-YB-13).

\section{REFERENCES}

[1] R.J. Pinnington, "Rubber friction on rough and smooth surfaces", Wear, vol. 267, pp. 1653-1664, 2009.

[2] C. Zhan, "Study on friction and wear property and lubricant mechanism of water lubricated bearing", Chongqing University Doctoral dissertation, pp. 72-89, 2003.

[3] T.A. Simpson and R.A. Ibrahim, "Nonlinear friction-induced vibration in water-lubricated bearings", Journal of Vibration and control, vol. 2, pp. 87-113, 1996.

[4] B. Bhushan, "Stick-slip induced noise generation in waterlubricated compliant rubber bearings", Journal of Lubrication Technology, vol. 102, pp. 201-210, 1980.
[5] H.T. Bans, J.B. Hood and N.G. Medhin and J.R. Samuels. "A stickslip/rouse hybrid model for viscoelasticity in polymers", Nonlinear Analysis: Real World Applications, vol. 9, no. 5, pp. 2128-2149, 2006.

[6] D.K. Baek, and M.M. Khonsari, "Fretting behavior of a rubber coating: friction characteristics of rubber debris", Wear, vol. 261, pp. 1114-1120, 2006.

[7] D.K. Baek, and M.M. Khonsari, "Friction and wear of a rubber coating in fretting", Wear, vol. 258, pp. 898-905, 2005.

[8] C. Dong, C. Yuan, Z. Liu, X. Yan, "Study on fatigue life evaluation of water lubricated rubber stern tube bearing", 2011 Prognostics \& System Health Management Conference, Shenzhen, China, 2011.

[9] R.L. Orndorff, Jr. "New UHMWPE/Rubber Bearing Alloy", Journal of Tribology, vol. 122, pp. 367-373, 2000.

[10] G.S. Chen, J.H. Lee, V. Narravula and T. Kitchin, "Friction and noise of rubber belt in low temperature condition: The influence of interfacial ice film", Cold Regions Science and Technology, vol. 71, pp. 95-101, 2012.

[11] E.-G. Peng, Z.-L. Liu, F. Lan, S.D. Zhang and M.C. Dai, "Research on noise generation mechanism of rubber material for waterlubricated bearings", Applied Mechanics and Materials, vol. 84-85, pp. 539-543, 2011.

[12] R.1. Leine, D.H. van Campen and W.J.G. Keultjes, "Stick-slip whirl interaction in drillstring dynamics", Journal of Vibration and Acoustics, vol. 124, pp. 209-220, 2002.

[13] A. Maccari, "Vibration control by nonlocal feedback and jerk dynamics", Nonlinear Dynamics, vol. 63, pp. 159-169, 2011.

[14] G.M. Rafferty, and D.T. Dutton, both of Jacksonville, Fla; W.Randall Tuchker, Oberlin. "Composite and fairwater structures for marine vessels", Ohio US005582528A 1996.

[15] L. Wang, X. Wang, Li Du, X. Zhang, B. Chen, and S.H. Hussain "A study on synthesis design and optimization analysis on scroll profiles for scroll vacuum pump", The Open Mechanical Engineering Journal, vol. 5, pp. 124-130, May 2011.

[16] G.X. Chen and Z.R. Zhou, "Time-frequency analysis of frictioninduced vibration under reciprocating sliding conditions", Wear, vol. 262, pp. 1-10, 2007.

[17] X. Wu, J. Wang, K. Xiao, and J. Zhu. "Dynamics Simulation of water lubricated bearing", Lubrication Engineering, vol.133, pp. 21-24, 2008.

[18] S. Merz, N. Kessissoglou, R. Kinns, and S. Marburg. "Minimisation of the sound power radiated by a submarine through optimisation of its resonance changer", Journal of Sound and Vibration, vol. 329, pp. 980-993, 2010.

[19] P. Wickramarachi and R. Singh, "Analysis of friction-induced vibration leading to eek noise in a dry friction clutch", The 2002 International Congress and Exposition on Noise Control Engineering, August 19-21, 2002.

[20] C. Zou, J. Wang, J. Yu and K. Xiao, "Effect of thickness and hardness of rubber underlayer on frictional coefficient of waterlubricated integer bearings", Lubrication Engineering, vol. 174, pp 40-43, 2006.

[21] C. Guangxiong and Z. Zhongrong, "Prediction of friction squeal using finite element method", Chinese Journal of Mechanical Engineering, vol. 43, no. 6, pp. 164-168, June 2007.

[22] Z. Chunliang, Y. Shiqin, L. Shunlong, Z. Hongtao and M. Yong, "Numerical simulation of longitudinal groove ship aft stern tube bearing fluid field", Lubrication Engineering, vol. 183, no. 11, pp. 39-44, 2006.

[23] B.N.J. Persson, "On the theory of rubber friction", Surface Science, Vol.401, pp. 445-454, 1998.

[24] J. J. Thomsen, "Using fast vibrations to quench friction-induced oscillations", Journal of Sound and Vibration, vol. 228, no. 5, pp. 1079-1102, 1999.

[25] B.N.J. Persson and A.I. Volokitin, "Rubber friction on smooth surfaces", The European Physical Journal E: Soft Matter and Biological Physics, vol. 21, no. 1, pp. 69-80, July 2006.

[26] N. Mihajlovic, N. van de Wouw, M.P.M. Hendriks and H. Nijmeijer, "Friction-induced vibration in an experimental drillstring system for various friction situations", in Proceedings of the ENOC-2005, Eindhoven, Netherlands, 7-12 August 2005.

[27] C. M. Jung and B. F. Feeny, "Friction-induced vibration in periodic linear elastic media", Journal of Sound and Vibration, vol. 252, no. 5, pp. 945-954, May 16, 2002.

[28] J.X. Wang, X. Hua, K. Xiao and J. Zhu, "Two-dimensional study of lubrication mechanism of water-lubricated rubber alloy bearing", 
International Journal of Computer Applications in Technology, vol. 40, no.1, pp. 98-106, 2011.

[29] X. Li, Z. Du, L. Xia, and J.X. Ding "Numerical simulation of the dynamic response of vessel mast structure under air explosion", Journal of Ship Mechanics, vol.10, pp. 151-158, 2006.
[30] Z. Liu, Principles of tribology, Peking: Higher Education Press, pp. 62-105, February 2009.

Received: June 11,2012

Revised: July 19, 2012

Accepted: July 19, 2012

(C) Peng et al.; Licensee Bentham Open.

This is an open access article licensed under the terms of the Creative Commons Attribution Non-Commercial License (http://creativecommons.org/licenses/by-nc/3.0/) which permits unrestricted, non-commercial use, distribution and reproduction in any medium, provided the work is properly cited. 